\title{
Bifurcations and multistability in the extended Hindmarsh-Rose neuronal oscillator
}

\author{
E.B. Megam Ngouonkadi a,*, H.B. Fotsin ${ }^{\text {a }}$, P. Louodop Fotso ${ }^{a, b}$, V. Kamdoum \\ Tamba $^{a}$, Hilda A. Cerdeira ${ }^{b}$ \\ a Laboratory of Electronics and Signal Processing, Department of Physics, Faculty of Sciences, University of Dschang, P.O. Box 067, \\ Dschang, Cameroon \\ ${ }^{\mathrm{b}}$ Instituto de Física Teórica, UNESP, Universidade Estadual Paulista, Rua Dr. Bento Teobaldo Ferraz 271,Bloco II, Barra Funda, São Paulo \\ 01140-070, Brazil
}

\section{A R T I C L E I N F O}

\section{Article history:}

Received 14 December 2015

Revised 29 January 2016

Accepted 2 February 2016

Available online 28 February 2016

\section{Keywords:}

Hopf bifurcation

Hindmarsh-Rose oscillator

Periodic solution

Bifurcation diagram

Multistability

Crisis

\begin{abstract}
A B S T R A C T
We report on the bifurcation analysis of an extended Hindmarsh-Rose (eHR) neuronal oscillator. We prove that Hopf bifurcation occurs in this system, when an appropriate chosen bifurcation parameter varies and reaches its critical value. Applying the normal form theory, we derive a formula to determine the direction of the Hopf bifurcation and the stability of bifurcating periodic flows. To observe this latter bifurcation and to illustrate its theoretical analysis, numerical simulations are performed. Hence, we present an explanation of the discontinuous behavior of the amplitude of the repetitive response as a function of system's parameters based on the presence of the subcritical unstable oscillations. Furthermore, the bifurcation structures of the system are studied, with special care on the effects of parameters associated with the slow current and the slower dynamical process. We find that the system presents diversity of bifurcations such as period-doubling, symmetry breaking, crises and reverse period-doubling, when the afore mentioned parameters are varied in tiny steps. The complexity of the bifurcation structures seems useful to understand how neurons encode information or how they respond to external stimuli. Furthermore, we find that the extended Hindmarsh-Rose model also presents the multistability of oscillatory and silent regimes for precise sets of its parameters. This phenomenon plays a practical role in short-term memory and appears to give an evolutionary advantage for neurons since they constitute part of multifunctional microcircuits such as central pattern generators.
\end{abstract}

(c) 2016 Elsevier Ltd. All rights reserved.

\section{Introduction}

Chaos can appear in systems of autonomous or non autonomous ordinary differential equations possessing few as three variable and one or two nonlinearities, since the pioneering work of Lorentz [1], Liu et al. [2] and Rössler

\footnotetext{
* Corresponding author. Tel.: +237 677440322.

E-mail address: elyemegambertrhand@yahoo.fr, megam.bertrand89@ gmail.com (E.B. Megam Ngouonkadi).
}

[3]. Over the years, many other chaotic systems have been discovered [4-8] and there have been many investigations on their dynamical behaviors [9-14]. For example, the building blocks of the central nervous system, neurons, are strongly complex dynamical systems. In order to understand the cell's intrinsic neurocomputational properties, much of present neuroscience research focusses on voltage- and second-messenger-gated currents in individual cells. It is commonly assumed that the knowledge of the currents is enough to find what the cell is doing, and why it is doing it. This is however in contradiction with the 
half-century-old observations, which state that cells having alike currents can yet provide quite different dynamics. In 1948, Hodgkin [15] found that, injecting a DC-current of different amplitude in isolated axons, results in the exhibit of repetitive spiking with different low frequencies and inhibition of responses in a narrow frequency band. Largely ignored by the neuroscience community, these observations were investigated a few decades later by Rinzel and Ermentrout [16]. They show that the observed behaviors are due to different bifurcation mechanisms of excitability. But, the model studied is the one introduced by Hodgkin and Huxley [9] (HH) which is rather complex and time consuming in numerical simulations. The extended Hindmarsh-Rose (eHR) neuronal oscillator is a simpler mathematical model, and it has been shown that it presents most of the HH's characteristics [17]. But from a nonlinear dynamical systems point of view, does the eHR neuronal oscillator behavior bring out how neurons respond to stimulus? Does the model present the multistability mechanism? Our aim is to bring some contribution to the field by studying this model in detail, and by examining these points.

The objective of mathematical models is to find the genuine trade off between accuracy and simplicity. The most important question in computational neuroscience is therefore, which characteristics of the complex dynamics are necessary to observe the specific tasks carried out by a neuron? In 1952, a mathematical model describing neuron activity was provided by Hodgkin and Huxley $(\mathrm{HH})$ [9]. During years, different other models have been developed and studied [18-20]. Here we focus, on the Hindmarsh-Rose (HR), neuronal oscillator [17,21-23], proposed by Hindmarsh and Rose in 1984, after the formulation of their 2-equations model [21]. Their main goal was to model synchronization of firing of two snail neurons in a simple way, without the use of the Hodgkin-Huxley $(\mathrm{HH})$ equations [22,24]. Hence, with the aim to create a neuron model that exhibits triggered firing, some modifications were done on the 2-equations model (by adding an adaptation variable, representing the slowly varying current, that changed the applied current to an effective applied one) to obtain the 3 -equations model $[21,24]$. This model has been very popular in studying biological properties of spiking and bursting neurons. A few years later, Selverston et al. [17], studied a computational and electronic model of stomatogastric ganglion (STG) neurons. They found during their analysis that, biological neurons could be modeled with only three or four degrees of freedom [17]. They focused themselves on the familiar 3-dimensional HR model, and discovered that, in spite of the fact that, this 3dimensional model can produce several modes of spikingbursting behaviors seen in biological neurons, its parameter space for chaotic activity is much more limited than observed in real neurons. That is why they proposed a modified version of this model, by adding a fourth term (a slower process) representing the calcium dynamics [17]. The system's complexity increases and it was then able to reproduce the complex dynamical (spiking, bursting and chaotic) behavior of pyloric central pattern generator neurons of the lobster stomatogastric system [17,25].
Over the last decades, some detailed investigations and studies of bifurcations and the dynamics of models such as $\mathrm{HH}$ model, Fitzhugh-Nagumo model, Izhikevich model or the third order HR model, have been done [19,26-28]. Particular attention has been devoted to the third order HR model in the cited articles, from which the transitions between quiescent asymptotic behaviors, continuous spiking regimes and global picture of the bifurcation scenario with respect to two parameters, with an outline to the effects of two further parameters have been obtained. Recall that, a better adjustment of the behavior of electronics neurons, when connected to its living counterpart, is represented by the fourth order HR model, compared to the third order model $[17,23]$. Several details of the shape of spiking-bursting activity, can also be adjusted with the help of this extended model. Furthermore, it presents more complex behavior than the third order model [17,23], and it can describe the calcium exchange between intracellular warehouse and the cytoplasm, to completely produce the chaotic behavior of the stomatogastric ganglion neurons $[17,23]$. Besides, the region of parameter space where chaos appears is larger than that of the three dimensional equations [29]. Hence, a bifurcation analysis of such model is important to understand transitions between stable bursting solutions and continuous spiking regimes, and the fold of cycles bifurcations cascade, rousing to transitions between quiescent asymptotic behaviors and bursting regimes, as in the third order model; our aim here is to bring some contribution by studying the dynamical behaviors of such model, which may be helpful in understanding how the calcium exchange is operated in the stomatogastric ganglion neurons. Thus, the first goal of our work, is to consider Hopf bifurcations of such a system by applying the normal form theory introduced by Hassard et al. [30]. Afterwards, we use a combination of bifurcation theory and numerical integration to investigate bifurcation points, where stable or unstable bifurcations occur in the system. Even if the HR model dates from 1984, and many dynamical studies are found in the literature [26,27,31-36], no theoretical analysis has been given for its extended model to the best of our knowledge.

Recall that, the most used neuron model for studying behavior of interacting neurons to understand afore problems, are the $\mathrm{HH}$ type models [9]. Its complexity needs expensive numerical time for the resolution of the differential equations. That is why, reduced models showing essentially equivalent dynamics such as the HR model presents good properties and are commonly used nowadays for this kind of analysis. In this manuscript, we focused on the latter described with the following system of differential equations $[17,23,29,37,38]$ :

$$
\left\{\begin{array}{l}
\dot{x}=a y+b x^{2}-c x^{3}-d z+I_{D C}, \\
\dot{y}=e-f x^{2}-y-g w, \\
\dot{z}=\mu[-z+s(x+h)], \\
\dot{w}=v[-k w+r(y+l)] .
\end{array}\right.
$$

Here, $a, b, c, d, e, f, g, \mu, s, h, v, k, r$ and $l$, are constants which express the current and conductance based dynamics. $I_{D C}$ represents the injected current. Notice that, this model is relevant since it reproduces the observed 
aspect of neuronal membrane voltage activity [17,23,37], while the complicated current-voltage relationships of the conductance-based model are replaced by polynomials. $x$ represents the membrane voltage, and $y$ a fast current. $z$ is a slow current since $\mu \ll 1$. $w$ is a slow dynamical process as $v<\mu \ll 1[17,23]$. The parameters $\mu$ and $v$ play a very important role in neuron activity; the first represents the ratio of time scales between fast and slow fluxes across the neuron's membrane and the second controls the speed of variation of the slower dynamical process $w$, particularly the calcium exchange between intracellular warehouse and the cytoplasm. They will be chosen together with the injected current as our bifurcation parameters.

In 1982, Arecchi et al. [39], investigated the scheming phenomenon of the coexistence of two attractors in a nonlinear system. They called this behavior, generalized multistability. It has also been found that multiple attractors appear in an ample variety of systems such as: mechanical systems, geophysical models and neuronal systems [36,40-43]. Such systems are very sensitive to parameters variation or external disturbances and particularly to noise which is inevitable in any natural or technical system. As an example, Pastor-Díaz and Lopez-Fraguas [44] analyze extensively the multistability properties of some dynamical systems. With the help of special tools as Poincaré maps and probability distribution functions, they found that the complex dynamics that occurred in such systems could be understood through simple discrete transformations [45]. They also found that chaotic synchronization regime can appear in a combined master-slave system from the coupled oscillators when some particular values of the control parameter are chosen [45]. Furthermore, multistability represents an essential inherent property of the dynamics of neurons and of neural networks [36,41-43,46-49]. It is important in understanding short-term memory and other neuronal behaviors. The coexistence of tonic spiking and silence and the one of tonic spiking and bursting, are mechanisms showing bistability, and are well understood [36,41-43,50-55]. Despite the works found in the literature, classification of mechanisms supporting multistability of oscillatory or silent regimes is still today a great problem to both, the theory of dynamical system and neuroscience. To the best of our knowledge, no investigations of such phenomena have been done in the extended Hindmarsh-Rose neuronal oscillator. Hence, the second goal of the present study is to show that multiple attractors can be found and coexist in this oscillator, and to make a contribution in the study of the dynamic behaviors of the latter.

Our study is organized as follows. In Section 2, we present the system's equilibria and their stability. With the help of the normal form theory, the direction of the Hopf bifurcation and the stability of the bifurcating periodic flows are studied in detail in Section 3. Section 3.2 presents numerical results obtained from the previous analytic studies. In the final part of this work (Section 4) we analyze some bifurcation diagrams according to parameters $I_{D C}, \mu$ and $v$ in which some comments on the birth of chaos are presented through numerical studies, followed with the coexistence of multiple attractors for the same parameter's settings. We discuss the main results of this paper and present some related future works in the conclusions (see Section 5).

\section{Stationary points and their stability}

\subsection{Stationary points}

Stationary points of a system are those points where the system's states do not depend on time. The nullclines (the curves along with $\dot{x}=0, \dot{y}=0, \dot{z}=0$ and $\dot{w}=0$, see Eq. (2) below), give information regarding both the steady states and the vector field of the system.

$\left\{\begin{array}{l}a y+b x^{2}-c x^{3}-d z+I_{D C}=0 \\ e-f x^{2}-y-g w=0 \\ \mu[-z+s(x+h)]=0 \\ v[-k w+r(y+l)]=0\end{array}\right.$

Their intersections give its steady states whose stability depends on its parameters. Combining different terms is Eq. (2) gives birth to two such nullclines defined by:

$y=\frac{c x^{3}+d s(x+h)-b x^{2}-I_{D C}}{a}=F(x)$,

and

$y=\frac{k}{k+g r}\left(e-f x^{2}-\frac{g r l}{k}\right)=G(x)$.

There may be 1, 2 or 3 steady states of the extended HR neuronal model depending upon the relation of $F(x)$ and $G(x)$ functions. Hence, setting $F(x)=G(x)$, yields the following third order polynomial for the $x$ state:

$x^{3}+A x^{2}+B x+D=0$,

where, $\quad A=-\frac{1}{c}\left[b-\frac{a f}{1+\frac{g r}{k}}\right], \quad B=\frac{d s}{c} \quad$ and $\quad D=-\frac{1}{c}\left[\left(e-\frac{g r l}{k}\right)\right.$ $\left.\frac{a}{1+\frac{g r}{k}}-d s h+I_{D C}\right]$. Let us define: $x=\xi-\frac{A}{3}$, after some mathematical manipulations Eq. (5) becomes:

$\xi^{3}+p \xi+q=0$,

(where, $p=B-\frac{A^{2}}{3}, q=D-\frac{1}{3} A B+\frac{2}{3} A^{3}$ ), from which solutions give the system's equilibria. Thus, in order for Eq. (6) to have 1, 2 or 3 real roots, the following conditions must hold:

1. If $4 p^{3}+27 q^{2}>0$ then our system has a unique equilibrium point $S_{e}=\left(x_{e}, y_{e}, z_{e}, w_{e}\right)$ given by,

$$
\left\{\begin{array}{l}
x_{e}=\left(-\frac{q}{2}+\left(\frac{q^{2}}{4}+\frac{p^{3}}{27}\right)^{\frac{1}{2}}\right)^{\frac{1}{3}}+\left(-\frac{q}{2}-\left(\frac{q^{2}}{4}+\frac{p^{3}}{27}\right)^{\frac{1}{2}}\right)^{\frac{1}{3}}-\frac{A}{3} \\
y_{e}=\frac{e-f x_{e}^{2}-\frac{g r l}{k}}{1+\frac{g r}{k}} \\
z_{e}=s\left(x_{e}+h\right) \\
w_{e}=\frac{r y_{e}+r l}{k}
\end{array}\right.
$$

2. If $4 p^{3}+27 q^{2}<0$, we have three fixed points,

$$
\left\{\begin{array}{l}
x_{e i}=2 \sqrt{-\frac{p}{3}} \cos \left[\frac{1}{3} \arccos \left(-\frac{q}{2} \sqrt{-\frac{27}{p^{3}}}\right)+\frac{2 i \pi}{3}\right]-\frac{A}{3}, \\
y_{e i}=\frac{e-f x_{e i}^{2}-\frac{g r l}{k}}{1+\frac{g r}{k}} \\
z_{e i}=s\left(x_{e i}+h\right), \\
w_{e i}=\frac{r y_{e i}+r l}{k}, \quad i=1,2,3 .
\end{array}\right.
$$

3. Finally, if $4 p^{3}+27 q^{2}=0$, we have two equilibrium points, one simple and one double, 

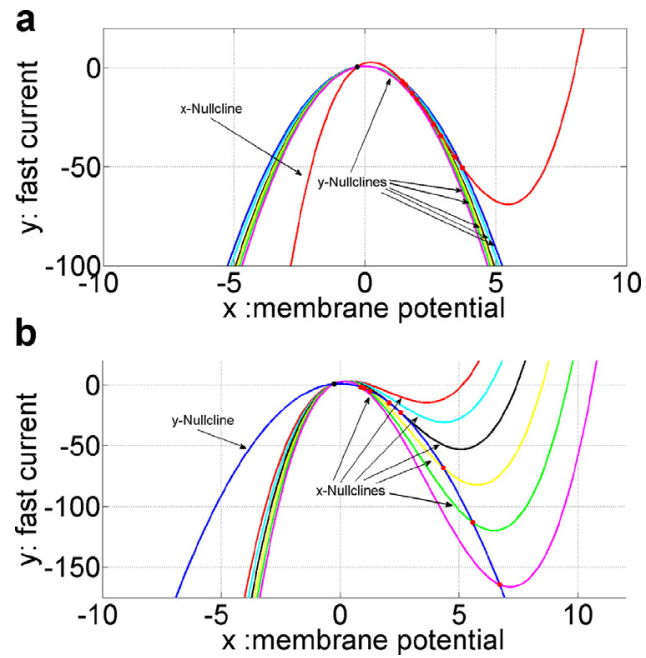

Fig. 1. Shifting the $y$-nullcline by changing $f$ while keeping other parameters constant (a) or shifting the $x$-nullcline by changing $b$ while keeping the other parameters constant (b), reduces the number of equilibria from 3 to 2 to 1 (a) or increases this number from 1 to 2 to 3 (b). The stability of each equilibrium is shown; red dots correspond to unstable equilibria while black dots correspond to stable equilibria (the other parameters are given in the text). (For interpretation of the references to color in this figure legend, the reader is referred to the web version of this article.)

$$
\left\{\begin{array}{l}
x_{e 1}=\frac{3 q}{p}-\frac{A}{3}, \\
x_{e 2}=x_{e 3}=-\frac{3 q}{2 p}-\frac{A}{3}, \\
y_{e i}=\frac{e-f x_{e i}^{2}-\frac{g r l}{k}}{1+\frac{g r l}{k}}, \\
z_{e i}=s\left(x_{e i}+h\right), \\
w_{e i}=\frac{r y_{e i}+r l}{k}, \quad i=1,2,3 .
\end{array}\right.
$$

To illustrate the above developments, we consider the following set of parameters: $a=1.0 ; c=1.0 ; d=0.99$ $e=1.01 ; \quad g=0.0278 ; s=3.966 ; \quad h=1.605 ; \quad k=0.9573$; $r=3.0 ; v=0.0009 ; l=1.619$ and $I_{D C}=3.99938$. Fig. $1(\mathrm{a})$ and (b) represent both nullclines for different values of parameter $f$ and $b$ respectively. More precisely, in Fig. 1(a) blue, cyan, black, yellow, green and magenta curves are plotted for $f=4.0 ; f=4.2 ; f=4.5 ; f=4.6$; $f=4.8$; and $f=5.0$, while in Fig. 1(b), red, cyan, black, yellow, green and magenta curves are plotted for $b=6.0$; $b=7.0 ; \quad b=8.0 ; \quad b=9.0 ; \quad b=10.0 ;$ and $b=11.0$, respectively. In these figures, nullclines intersections are given by black and red dots. We observe that varying the $f$ value ( $b$ value in Fig. 1(b)) shifts the $y$-nullcline ( $x$-nullcline), whose effect is to reduce (increase) the number of equilibria as classified in the previous conditions (conditions 1,2 and 3 ), from 3 to 1 (1 to 3 ). For example for $f=4.5$ we have 3 equilibria given by: $\left\{S_{e}=\right.$ $(1.813459312,-12.81358363,13.55760963,-35.08174125)$, (2.907258884, -34.187333944, 17.89561874, -102.0631133), $(-0.2850955384,0.4628698494,5.234741095,6.524192571)\}$, obtained when condition 2 is satisfied.

\subsection{Fixed points stability}

In order to analyze the steady states stability and the local bifurcations susceptible to occur in system (1) when varying the parameters, we linearize this system around these equilibria $\left(x_{e}, y_{e}, z_{e}, w_{e}\right)$ and the following $4 \times 4$ Jacobian matrix is obtained:

$\mathbf{J}_{\mathbf{e}}=\frac{\partial f^{i}}{\partial x_{j}}\left(x_{e}\right)=\left(\begin{array}{cccc}\vartheta & a & -d & 0 \\ -2 f x_{e} & -1 & 0 & -g \\ \mu s & 0 & -\mu & 0 \\ 0 & v r & 0 & -k v\end{array}\right)$,

where $\vartheta=2 b x_{e}-3 c x_{e}^{2}$. Coming back to Fig. 1, we observe that shifting the $x$ or $y$ nullcline reduces or increases the number of equilibria from 3 to 1 or from 1 to 3 respectively. As $f$ (respectively $b$ ) is increased, the $y$-nullcline (respectively the $x$-nullcline) is shifted down (Fig. 1(a) and (b)). A bifurcation results in the system when the two equilibria marked by red dots coalesce and then disappear into a saddle-node or fold bifurcation (Fig. 1(a)). To illustrate this by numerical results, we consider $b=8.575$ and $f=4.5$, the other parameters kept unchanged. The eigenvalues of the first equilibrium $\left(S_{e 1}=\right.$ $(1.813459312,-12.81358363,13.55760963,-35.08174125))$ are: $\quad \lambda_{1}=20.4745 ; \quad \lambda_{2}=-0.240961285112409 ; \quad \lambda_{3}=$ $-0.000806879431116+0.000534667614844 i ; \quad \lambda_{4}=$ $-0.000806879431116-0.000534667614844 i$, where $i$ denotes the unit of the imaginary number. Since $\lambda_{1}$ is a real positive number, $\lambda_{2}$ a real negative number, $\lambda_{3}$ and $\lambda_{4}$ a pair of complexes conjugate numbers, this equilibrium is an unstable saddle-focus. For the second equilibrium $S_{e 2}=$ (2.907258884,-34.187333944, 17.89561874,-102.0631133), we obtain $\lambda_{1}=0.07494944705, \lambda_{2}=23.43170929, \lambda_{3}=$ $-0.0006703607107, \quad \lambda_{4}=-0.005972751298$ for which there are two positive and two negative real values; thus we conclude that it is unstable. Considering the third equilibrium $S_{e 3}=(-0.2850955384,0.4628698494$, $5.234741095,6.524192571)$, its corresponding eigenvalues are: $\quad \lambda_{1}=-0.0009567223136, \quad \lambda_{2}=-0.005519762104$, $\lambda_{3}=-0.4497626669$ and $\lambda_{4}=-5.679999301$. Its is a stable focus for the fact that, all the eigenvalues are negative real values.

Furthermore, the stability of the above equilibrium points and the type of bifurcation occurring at these points are given in a first point of view by looking at the solution of the characteristic equation of matrix $J_{e}$ :

$\varsigma^{4}+a_{1} \varsigma^{3}+a_{2} \varsigma^{2}+a_{3} \varsigma+a_{4}=0$,

where,

$a_{1}=k v+\mu+1-\vartheta$

$a_{2}=v r g+k v \mu+k v+\mu s d+\mu+2 f x_{e} a-(\mu+1+k v) \vartheta$

$a_{3}=v r g \mu+k v \mu s d+k v \mu+2 k v a f x_{e}+\mu s d+2 \mu f_{a x}$ $-\vartheta(v r g+k v \mu+k v+\mu)$,

$a_{4}=v r g \mu s d+k v \mu s d+2 k v \mu f a x_{e}-\vartheta \mu(k v+v r g)$

In Fig. 2, we show these eigenvalues in the complex plane $(\operatorname{Re}(\varsigma)$, Im $(\varsigma))$. Eq. (10) is solved using the NewtonRaphson method for the following ranges of parameters; $0 \leq \mu \leq 1.0$, keeping the others constant: $a=1.0 ; c=$ $1.0 ; d=0.99 ; \quad e=1.01 ; g=0.0278 ; s=3.966 ; h=1.605$; $k=0.9573 ; r=3.0 ; v=0.0009$ and $l=1.619$. Fig. 2(a) correspond to $b=8.575$ and $f=4.5$, while Fig. 2(b) correspond to $b=8.0$ and $f=4.0$. From these figures we can deduce some points regarding the stability of the equilibria and the bifurcation liable to appear in the extended 
a

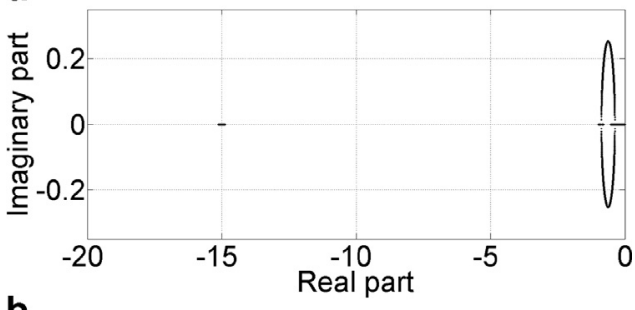

b

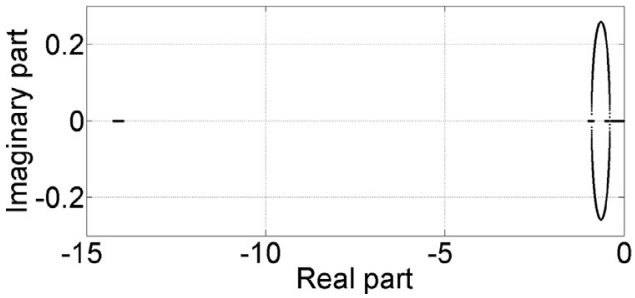

Fig. 2. Representation of the eigenvalues, solutions of the characteristic equation (10), in the complex plane $(\operatorname{Re}(\varsigma)$, Im $(\varsigma))$. The other parameters' values are defined in the text. (a) When $f$ is fixed to 4.5 ; (b) When $b$ is fixed to 8.0 .

Hindmarsh-Rose neuronal oscillator. The following conclusion can be made, the matrix $J_{e}$ is obviously real; hence, the observed symmetry throughout the real axis is the result of the appearance of complex conjugate pairs of its eigenvalues. Consequently, volumes in phase space are contracted if the real parts of the eigenvalues $(\varsigma)$ are all negative and expanded if they are positive. Contraction or expansion of spirals is observed for complex eigenvalues of the Jacobian matrix [56,60]. The equilibrium is a saddle, if the complex eigenvalues have real parts of different signs. If the eigenvalues are complex conjugate with zero real part, we have a center.

Fig. 3 also helps to understand the effect of varying the parameters $b$ or $f$ and how the location, number and stability of the equilibria change when these parameters are varied. We can clearly depict from these figures the bifurcation points and the kind of bifurcations occurring in our system. Blue dots show that the equilibrium is stable while red dots show that it is unstable. In each of these figures, we see 3 qualitative changes (i.e. bifurcations) as both $f$ (Fig. 3(a)) and $b$ (Fig. 3(b) and (c)) are varied. For example, at point "bifurcation3" in Fig. 3(a), a saddle-node bifurcation occurs since we observed a coalescence and a disappearance of two equilibria. We observe in Fig. 3(b) that at the point marked by "bifurcation1", the equilibrium switches stability giving birth to a transcritical bifurcation. These bifurcations correspond to those obtained in previous paragraphs.

\section{Local stability, existence and direction of Hopf bifurcation.}

\subsection{Theoretical analysis}

What happens when a pair of complex-conjugate characteristic exponents of an equilibrium state crosses over the imaginary axis, is the main objective of this section. The drain of stability in this case is directly connected to a
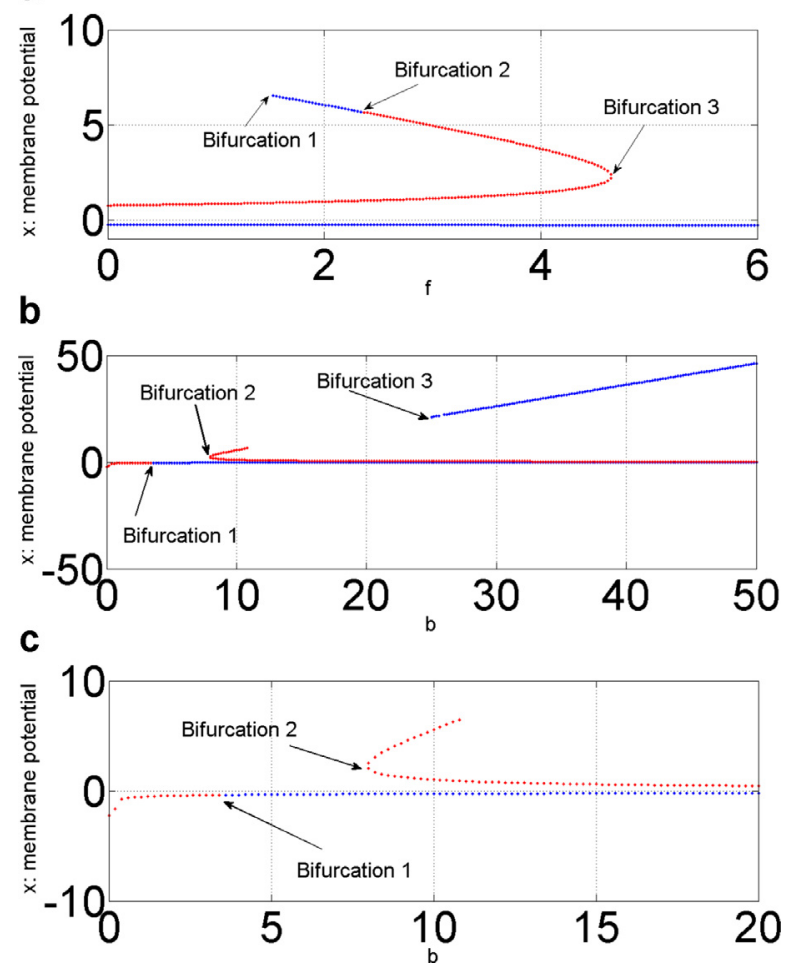

Fig. 3. Membrane potential as a function of $f(a)$ and $b$ (b). Its helps to understand how the location, number and stability of the equilibria change when the parameters of the system are varied. This is done by finding the bifurcation points of the system. We observe three qualitative changes as $f(a)$ or $b(\mathrm{~b})$ is varied i.e. 3 bifurcations. (For interpretation of the references to color in this figure legend, the reader is referred to the web version of this article.)

the disappearance or the birth of a periodic orbit. This bifurcation (known as the Hopf bifurcation) represents the merest mechanism for transition from a stationary regime to oscillations (or from oscillations to a stationary regime) and can highlight proper interpretation of numerous physical phenomena. That is why it usually plays a special role in the theory of bifurcations. For these purposes, we consider the Jacobian matrix of the linearized system around the equilibria $S_{e}$ given in the previous subsection. Its characteristic equation is described by Eq. (10) given in Section 2.2. To guarantee the existence of the Hopf bifurcation in system (1) and the satisfaction of the transversality condition [66], we consider the derivative of the characteristic equation (Eq. (10)) with respect to $\mu$ (considered as the control parameter)

$$
\begin{aligned}
\frac{\partial f(\mu)}{\partial \mu}= & 4 \varsigma^{4}(\mu) \frac{\partial \varsigma(\mu)}{\partial \mu}+\frac{\partial a_{1}(\mu)}{\partial \mu} \varsigma^{3}(\mu) \\
& +3 a_{1}(\mu) \varsigma^{2}(\mu) \frac{\partial \varsigma(\mu)}{\partial \mu}+\frac{a_{2}(\mu)}{\partial \mu} \varsigma^{2}(\mu) \\
& +2 a_{2}(\mu) \varsigma(\mu) \frac{\partial \varsigma(\mu)}{\partial \mu}+\frac{\partial a_{3}(\mu)}{\partial \mu} \varsigma(\mu) \\
& +a_{3}(\mu) \frac{\partial \varsigma(\mu)}{\partial \mu}+\frac{\partial a_{4}(\mu)}{\partial \mu}=0 .
\end{aligned}
$$


For these purposes we assume the eigenvalues to be complex: $\varsigma(\mu)=\alpha(\mu)+i \omega(\mu)$. Thus, solving $\frac{\partial f}{\partial \mu}\left(\mu_{c}\right)=0$ and separating imaginary and real parts gives

$\frac{\partial \alpha\left(\mu_{c}\right)}{\partial \mu}=-\left(\frac{\hat{a}_{0} \hat{b}_{0}+\hat{a}_{1} \hat{b}_{1}}{\hat{a}_{0}^{2}+\hat{b}_{1}^{2}}\right)$,

and

$\frac{\partial \omega\left(\mu_{c}\right)}{\partial \mu}=-\left(\frac{\hat{a}_{0} \hat{a}_{1}+\hat{b}_{0} \hat{b}_{1}}{\hat{a}_{0}^{2}+\hat{b}_{1}^{2}}\right)$,

where $\hat{a}_{0}=\left(4\left(3 \alpha^{2}-\omega^{3}\right)+6 \alpha a_{1} \omega+2 a_{2} \omega\right), \hat{b}_{0}=\left(\left(3 \alpha^{2} \omega-\right.\right.$ $\left.\left.\omega^{3}\right) \frac{\partial a_{1}}{\partial \mu}+2 \alpha \omega \frac{\partial a_{2}}{\partial \mu}+\omega \frac{\partial a_{3}}{\partial \mu}\right), \quad \hat{a}_{1}=\left(\left(\alpha^{3}-3 \alpha \omega^{2}\right) \frac{\partial a_{1}}{\partial \mu}+\left(\alpha^{2}-\right.\right.$ $\left.\left.\omega^{2}\right) \frac{\partial a_{2}}{\partial \mu}\right), \quad \hat{b}_{1}=\left(4\left(\alpha^{3}-3 \alpha \omega^{2}\right)+3\left(\alpha^{2}-\omega^{2}\right)+2 a_{2} \alpha+a_{3}\right)$. Examining these relations, we see that $\frac{\partial \alpha\left(\mu_{c}\right)}{\partial \mu} \neq 0$. Under the restriction that $\Re\left(S_{j}\left(\mu_{c}\right)\right)<0$ for $j=3,4$, the Poincaré-Andronov-Hopf theorem holds and a Hopf bifurcation can occur at $\left(S_{e}, \mu_{c}\right)$ of system (1).

Let us derive now a relation between the system's parameters corresponding to this bifurcation around the equilibrium $S_{e}$. To attend our aim, we substitute $\varsigma=i \omega_{0}$ into the characteristic equation (10) and obtain: $\left(\omega_{0}^{4}-\right.$ $\left.a_{2} \omega_{0}+a_{4}\right)+i\left(a_{3} \omega_{0}+\omega_{0}^{3} a_{1}\right)=0$. From where, we conclude that such bifurcation occurs in the system when the following equations are verified

$\omega_{0}=\sqrt{\frac{a_{3}}{a_{1}}}$

and

$a_{3}^{2}-a_{1} a_{2} a_{3}+a_{1}^{2} a_{4}=0$.

We replace in Eq. (15) $a_{1}, a_{2}, a_{3}$ and $a_{4}$ by their expressions described in Section 2.2. After some mathematical manipulations, we derive the following equation from which solutions give the control parameter's values $(\bar{A}, \bar{B}$, $\bar{C}$ and $\bar{D}$ are described in the Appendix A Eqs. (A.1-A.4))

$\bar{A} \mu^{3}+\bar{B} \mu^{2}+\bar{C} \mu+\bar{D}=0$.

With the help of the Hassard et al. method, [30,59, $70,71]$ we now study the stability and period of the above Hopf bifurcation occurring at $\mu_{c}$. We suppose the existence of a conjugate pair of eigenvalues of the Jacobian matrix such that: $\alpha(0)=0$ and let $\omega_{0} \equiv \omega(0)>0$. We also suppose the existence of a similarity transformation $P$ which reduces the Jacobian $J_{e}\left(\mu_{c}\right)$ to the form:

$\mathbf{P}^{-1} \mathbf{J}_{\mathbf{e}}\left(\mu_{\mathbf{c}}\right) \mathbf{P}=\left(\begin{array}{ccc}\left(\begin{array}{cc}0 & -\omega_{0} \\ \omega_{0} & 0\end{array}\right) & 0 \\ & 0 & D\end{array}\right)$,

where $D$ is diagonal. The first two columns of $P$ represent the real and imaginary parts of the eigenvector corresponding to the eigenvalue $i \omega_{0}$ and, the eigenvectors corresponding to the real eigenvalues constitute the third and the fourth columns. In our case, the eigenvalues of the Jacobian matrix are given by $\varsigma_{1}=-\varsigma_{2}=i \omega_{0}$, $\varsigma_{3}=\frac{-a_{1}-\sqrt{a_{1}^{2}+\frac{4 a_{1} a_{4}}{a_{3}}}}{2}$, and $\varsigma_{4}=\frac{-a_{1}+\sqrt{a_{1}^{2}+\frac{4 a_{1} a_{4}}{a_{3}}}}{2}$, and their associated eigenvectors are described in the Appendix (see Eqs. (A.5-A.8)). Thus, the matrix $P$ is defined as follows

$P=\left(\Re\left(v_{1}\right), \Im\left(v_{1}\right), v_{3}, v_{4}\right)=\left(p_{i j}\right)_{1 \leq i, j \leq 4}$.
Let us substitute $Y=P^{-1}\left(X-S_{e}\right)$, and $\tilde{F}(Y, \mu)=P^{-1} F$ $\left(P Y+S_{e}, \mu\right)$ in system (1), where $F(X, \mu)$ represents the vector field of such system, $X=(x, y, z, w)$ its states and $S_{e}=\left(x_{e}, y_{e}, z_{e}, w_{e}\right)$ its equilibria. We obtain after some manipulations

$\dot{Y}=\tilde{F}(Y, \mu)$.

Notice that, the stationary point of Eq. (18) is the origin. We demonstrate the existence of small amplitude periodic solutions of Eq. (18), for small $\mu$ in the case $\alpha^{\prime}(0) \neq 0, D_{11}$ $<0$ and $D_{22}<0\left(D_{11}\right.$ and $D_{22}$ are diagonal elements of the matrix $D$ ). To attend our objective, we follow firstly the procedures described by Hassard et al. [30,59,71], calculate and obtain the quantities described below at $\mu=\mu_{c}$ :

$F_{20}^{1}=\frac{p_{11}^{2}}{M}\left(p_{11}^{-1}\left(2 b-6 c x_{e}\right)-2 f p_{12}^{-1}\right) ;$

$F_{02}^{1}=\frac{p_{12}^{2}}{M}\left(p_{11}^{-1}\left(2 b-6 c x_{e}\right)-2 f p_{12}^{-1}\right) ;$

$F_{11}^{2}=-\frac{p_{11} p_{12}}{M}\left(p_{21}^{-1}\left(2 b-6 c x_{e}\right)-2 f p_{22}^{-1}\right)$

$F_{20}^{2}=-\frac{p_{11}^{2}}{M}\left(p_{21}^{-1}\left(2 b-6 c x_{e}\right)-2 f p_{22}^{-1}\right)$

$F_{02}^{2}=-\frac{p_{21}^{2}}{M}\left(p_{21}^{-1}\left(2 b-6 c x_{e}\right)-2 f p_{22}^{-1}\right) ;$

$F_{11}^{1}=\frac{p_{11} p_{12}}{M}\left(p_{11}^{-1}\left(2 b-6 c x_{e}\right)-2 f p_{12}^{-1}\right) ;$

$F_{30}^{1}=-\frac{6 p_{11}^{-1} p_{11}^{3} c}{M} ; \quad F_{12}^{1}=-\frac{6 p_{11}^{-1} p_{11} p_{12}^{2} c}{M} ;$

$F_{21}^{2}=\frac{6 p_{21}^{-1} p_{11}^{2} p_{12} c}{M} ; \quad F_{03}^{2}=\frac{6 p_{21}^{-1} p_{12}^{3} c}{M} ;$

$F_{30}^{2}=\frac{6 p_{21}^{-1} p_{11}^{3} c}{M} ; \quad F_{12}^{2}=\frac{6 p_{21}^{-1} p_{11} p_{12}^{2} c}{M} ;$

$F_{21}^{1}=-\frac{6 p_{11}^{-1} p_{11}^{2} p_{12} c}{M} ; \quad F_{03}^{1}=-\frac{6 p_{11}^{-1} p_{12}^{3} c}{M} ;$

$g_{20}=\frac{1}{4}\left[F_{20}^{1}-F_{02}^{1}+2 F_{11}^{2}+i\left(F_{20}^{2}-F_{02}^{2}-2 F_{11}^{1}\right)\right] ;$

$g_{11}=\frac{1}{4}\left[F_{20}^{1}+F_{02}^{1}+i\left(F_{20}^{2}+F_{02}^{2}\right)\right] ;$

$g_{02}=\frac{1}{4}\left[F_{20}^{1}-F_{02}^{1}-2 F_{11}^{2}+i\left(F_{20}^{2}-F_{02}^{2}+2 F_{11}^{1}\right)\right]$

$g_{21}=\frac{1}{8}\left[F_{30}^{1}+F_{12}^{1}+F_{21}^{2}+F_{03}^{2}+i\left(F_{30}^{2}+F_{12}^{2}-F_{21}^{1}-F_{03}^{1}\right)\right]$;

since $F_{10}^{1,1}=F_{01}^{2,1}=F_{10}^{2,1}=F_{01}^{1,1}=0$. Thus,

$\tilde{c}_{1}(0)=\frac{i}{2 \omega_{0}}\left(g_{20} g_{11}-2 g_{11} \bar{g}_{11}-\frac{1}{3} g_{02} \bar{g}_{02}\right)+\frac{g_{21}}{2}$

Finally we obtain the main results below, where:

$\mu_{2}=-\frac{\Re\left(\tilde{c}_{1}\right)}{\frac{\partial \alpha}{\partial \mu}\left(\mu_{c}\right)}, \quad \tau_{2}=-\frac{-\Im\left(\tilde{c}_{1}\right)+\mu_{2} \frac{\partial \omega}{\partial \mu}\left(\mu_{c}\right)}{\omega_{0}}$,

$\beta_{2}=2 \Re\left(\tilde{c}_{1}\right)$.

Results:

Taking into consideration the fact that, system (1) undergoes a Hopf bifurcation at equilibrium $S_{e}$ when parameter $\mu$ passes the value $\mu_{c}$, the following properties hold: 
(1) if $\mu_{2}<0\left(\mu_{2}>0\right)$, the Hopf bifurcation is supercritical (subcritical),

(2) if $\beta_{2}<0\left(\beta_{2}>0\right)$, the bifurcating periodic solutions are orbitally stable (unstable),

(3) the period of bifurcating periodic solutions increases (decreases), if $\tau_{2}>0\left(\tau_{2}<0\right)$.

From these results we conclude that, the system has a unique amplitude solution of approximated period

$T(\mu)=\frac{2 \pi}{\omega_{0}}\left(1+\tau_{2} \varrho^{2}+\mathcal{O}\left(\varrho^{4}\right)\right)$,

and a characteristic multiplier (or characteristic exponent) with largest real part associated with solutions

$\beta=\beta_{2} \varrho^{2}+\mathcal{O}\left(\varrho^{4}\right)$.

In which $\varrho^{2}=\left(\frac{\mu-\mu_{c}}{\mu_{2}}\right)+\mathcal{O}\left(\mu-\mu_{c}\right)^{2}$ (under the condition $\left.\mu_{2} \neq 0\right)$. The periodic solutions themselves are approximated by:

$X=X_{e}\left(\mu_{c}\right)+\left(\frac{\mu-\mu_{c}}{\mu_{2}}\right)^{1 / 2} \Re\left[e^{2 \pi i t / T(\mu)} v_{1}\right]+\mathcal{O}\left(\mu-\mu_{c}\right)$.

Where, $v_{1}$ represents the eigenvector of the Jacobian matrix at $\mu=\mu_{c}$, corresponding to the eigenvalue $\varsigma_{1}\left(\mu_{c}\right)$.

\subsection{Numerical results}

In order to illustrate the above theoretical results on the Hopf bifurcation, numerical computations are done. For this instance, we consider the system (1) with the following parameter values $a=1.0 ; b=3.0 ; c=1.0 ; d=$ $0.99 ; \quad e=1.01 ; f=5.0128 ; g=0.0278 ; \quad s=3.966 ; \quad h=$ $1.605 ; v=0.0009 ; k=0.9573 ; r=3.0, l=1.619$ and $I_{D C}=$ 3.024972, from where we obtain the unique equilibrium: $x_{e}=-0.7553399395, y_{e}=-1.831483449, \quad z_{e}=3.3697518$ and $w_{e}=-0.6658835764$. Considering this equilibrium, the critical values of the bifurcation parameter $\mu$ defined as solution of Eq. (16) are: $\mu_{c 1}=0.0002578485593, \mu_{c 2}=$ 0.1230628577 and $\mu_{c 3}=-7.0150113$. Since the parameter $\mu$ is always positive, we omitted the latter value in our simulation; and for convenience, only the second parameter is chosen and the same conclusion can be made when the first value is taken into account.

Therefore, considering the second critical value, we have two real eigenvalues $\varsigma_{3}\left(\mu_{c}\right)=-0.1153794092 .10^{-2}$ and $\varsigma_{4}\left(\mu_{c}\right)=-7.366425543$ and two pure imaginary ones $\varsigma_{1,2}\left(\mu_{c}\right)= \pm i 0.2084537603$. Moreover, $\frac{\partial \alpha\left(\mu_{c}\right)}{\partial \mu} \approx-0.7400902374 \neq 0$ and we can conclude from the previous section that the transversality condition is satisfied. The numerical computation of matrix $P$ and its inverse $P^{-1}$ are gives in the Appendix A. Furthermore, the various useful coefficients for the direction of the bifurcation are:

$g_{02}=0.062406309+3.2015034 i ; g_{11}=0.0624063096-$ $3.3253896 i ; \quad g_{20}=0.0624063096+3.2015034 i ; \quad g_{21}=$ $-0.1135724325-0.041342868 i$ and $\tilde{c}_{1}(0)=$ $-0.0382418625-35.74063142 i$. We finally compute:

$\mu_{2}=-0.057116142<0 ; \quad \beta_{2}=-0.076483725<0 \quad$ and $\tau_{2}=58.820075638>0$.
In the light of the above results, the Hopf bifurcation is supercritical. Since $\mu_{2}<0$, the solutions exist for $\mu<$ $\mu_{c}$ describing the direction of bifurcation. Furthermore, $\beta_{2}$ $<0$ and we conclude that the solutions are asymptotically stable orbits with an increasing period given approximatively by:

$$
\begin{aligned}
T= & 30.141865998(1-1029.832784(\mu-0.1230628577) \\
& \left.+\mathcal{O}(\mu-0.1230628577)^{2}\right) .
\end{aligned}
$$

The corresponding characteristic multiplier is:

$$
\begin{aligned}
\beta= & 1.33909123(\mu-0.1230628577) \\
& +\mathcal{O}(\mu-0.1230628577)^{2}
\end{aligned}
$$

and the periodic solutions are approximated by

$$
\begin{aligned}
X= & \left(\begin{array}{c}
-0.7553399395 \\
-1.831483449 \\
3.3697518 \\
-0.6658835764
\end{array}\right)+\left(\frac{\mu-0.1230628577}{-0.057116142}\right)^{1 / 2} \\
& \times \Re\left[e^{2 \pi i t / T(\mu)}\left(\begin{array}{c}
7.258414757-1.510428036 i \\
1.025009947-1.736244239 i \\
0.019952074+0.093932245
\end{array}\right)\right] \\
& +\mathcal{O}(\mu-0.1230628577) .
\end{aligned}
$$

Fig. 4 presents a family of periodic solutions, bifurcating out from equilibria. When $\mu$ decreases, the period of the bifurcating solutions decreases. This can be justified by the fact that the period of the solution in Fig. 4(c) is smaller than the one of Fig. 4(d), corresponding respectively to $\mu=$ 0.10 and $\mu=0.12$. Furthermore, each periodic solution is a stable limit cycle.

\section{Bifurcation and coexistence of multiple attractors}

\subsection{Bifurcation and birth of chaos}

The evolution of the asymptotic behavior of solutions are shown through the bifurcation diagram, as a function of a single parameter. We focused on those bifurcation parameters which can be manipulated experimentally, for example, $\mu, v$ and the injected current $I_{D C}$ in system (1).

In Fig. 5 we present the bifurcation diagram with respect to $I_{D C}$. The type of dynamical behavior of the model can be classified by examining the above diagram and its corresponding maximal Lyapunov exponent [61]. For this purpose, we solve Eq. (1) by the fourth-order Runge-Kutta method. The system is integrated for each value of the control parameter and for a sufficiently long time, after which transient is discarded. The computations were done using constant and variable parameters in extended mode and the time step is considered as $\Delta t<0.05$. For these plots, the following parameters' values were considered: $a=1.0 ; b=3.0 ; c=1.0 ; d=0.99 ; e=1.01 ; f=5.0128$; $g=0.0278 ; s=3.966 ; h=1.605 ; v=0.0009 ; k=0.9573$; $\mu=0.00215 ; r=3.0$ and $l=1.619$. The bifurcation diagrams are obtained by considering the minima of the membrane potential $x(t)$.

Notice that, the same conclusions as the ones below could have been made if the interspike intervals or the 
a

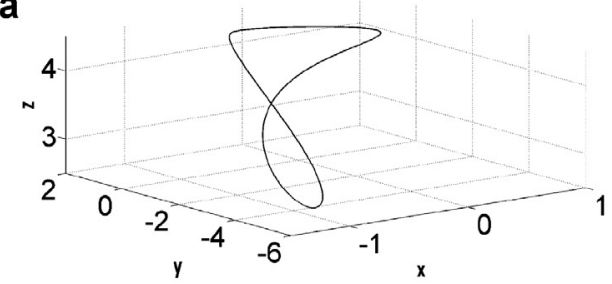

b

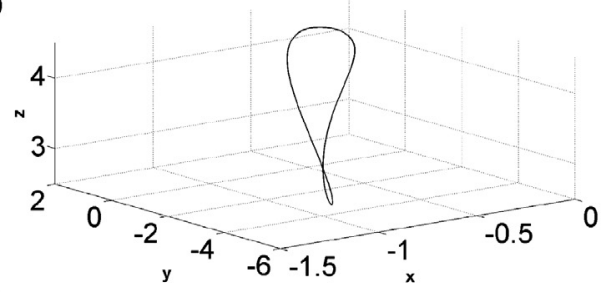

C

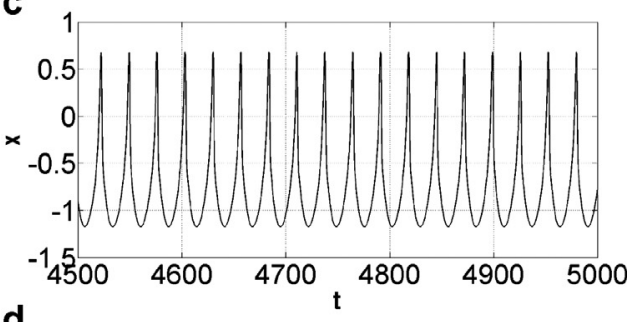

d

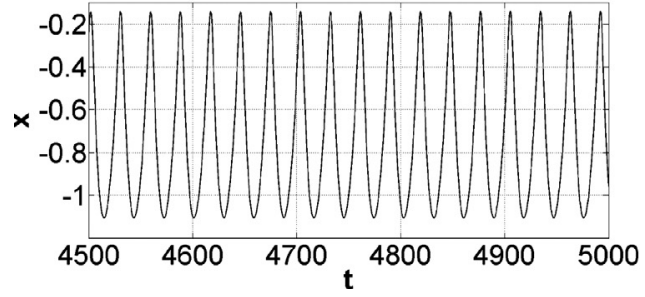

Fig. 4. Phase portrait and time series of system (1) with the parameters fixed in the text. (a) and (c) for $\mu=0.10<\mu_{c}$; (b) and (d) for $\mu=0.12<$ $\mu_{c}$. The asymptotic solution is a stable limit cycle.

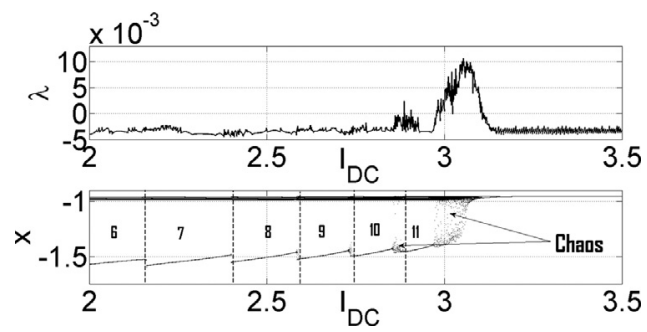

Fig. 5. Bifurcation diagram showing the coordinate $x(t)$ and the corresponding graph of the maximal Lyapunov exponent versus $I_{D C}$. The other parameters' values are defined in the text.

times of crossing of a surface in the phase space were considered. If $\lambda_{\max }<0$, the system evolves to a regular state. If $\lambda_{\max }=0$, it evolves into a torus state. Chaotic state is observed when $\lambda_{\max }>0$. Hence, when looking at this figure, one can observe different transitions to the chaotic state such as period doubling or crisis scenarios. For $I_{D C}=$ 3.0249, the corresponding maximal Lyapunov exponent is $\lambda_{\max }=0.000327$. Since it is positive, we conclude that the model behaves chaotically [37].

As it was found by Gonzales-Miranda $[27,28,62]$ for the original HR model, this model presents complex structures such as continuous crisis and block structured dynamics. A slender change in the bifurcation diagram shows up a continuous crisis at $I_{D C} \approx 2.978$ (see Fig. 5). This interior crisis is characterized by the transition between the spiking regime and the bursting regime [57,63-66]. We obtain bifurcation structure of an inverted period doubling cascade at the right hand side of the crisis domain (see Fig. 5). Spiking dynamics characterized by an irregular firing of spikes like the one in Fig. 6 are found in the region where the bifurcation diagram is very close. As we have said, block structure is found in the extended HR model, when $I_{D C}, \mu$ or $v$ are taken as bifurcation parameters (see Figs. 5 and 7). This behavior can be characterized in blocks by the periodicity $\eta$ of the flow. We can define each block with an interval of $I_{D C}, \mu$ or $v$ respectively, where the flows have the same period. For example, when $I_{D C} \in[1,1.212]$ the flows have periodicity 3 also called a burster with three spikes per burst, and more information about the other intervals are given in Fig. 5. We observe that chaotic regions can also be found within these blocks. The time series describing each block of Fig. 5 are illustrated in Fig. 6(a)-(f), where period 1, 3, 4, 6 and 9 (bursters with 1, 3, 4, 6 and 9 spikes per burst) and chaotic flows are found. They present the bursting and spiking activities of the extended HR model using the above parameters. As we can observe, there are two distinct time scales of oscillations in the system for some values of the injected current. In the phase space, the trajectories move slowly in one region and fast in the other region. These two regions are separated by the $y-z$ plane approximately along the firing threshold $x=-1$. Different time scales oscillations such as slow motion, as the membrane potential is flowing below the firing threshold, and fast motion when it oscillates above the threshold, are observed in the voltage traces in the above figures. Moreover, the complexity included by the fourth dynamical variable $w$ can be observed in the three dimensional phase space $(x, z, w)$ presented in Fig. $6(\mathrm{~g})$, and obtained for $I_{D C}=3.0249$.

The bifurcation diagram versus parameters $\mu$ and $v$ are given in Fig. 7(a) and (b), with $\mu \in[0.0,0.055]$ and $v \in[0.0,0.055]$. Notice that we superimposed two diagrams in these figures. In the diagram shown in red color, we considered the final state at each iteration of the control parameter, as the initial state for the next iteration. For the one in blue color, the initial state for all iterations of the control parameter were constant and taken as $\left(x_{e}=0.3, y_{e}=0.3 ; z_{e}=3.0, w_{e}=0.01\right)$. In these figures, we observe that there exists some value of the control parameter for which the neuron behavior changes abruptly. These changes are called crisis [57,63-66]. Other types of bifurcation structures such as period doubling and saddle-node bifurcation can also be seen, when the control parameters vary [66]. A zoom of some regions of Fig. 7(a) and (b) illustrates these observations (see Fig. 7(c) and (d), respectively). More complex asymptotic behaviors such as period doubling (PD) cascade, reverse period doubling (RPD), interior and exterior crisis (IC and 


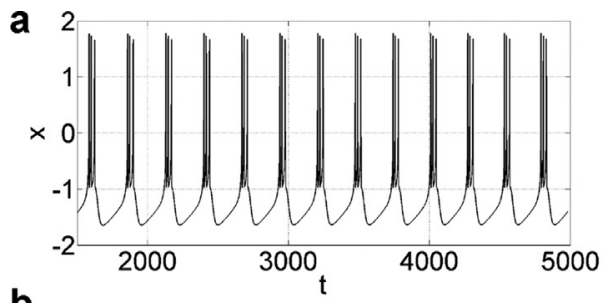

b
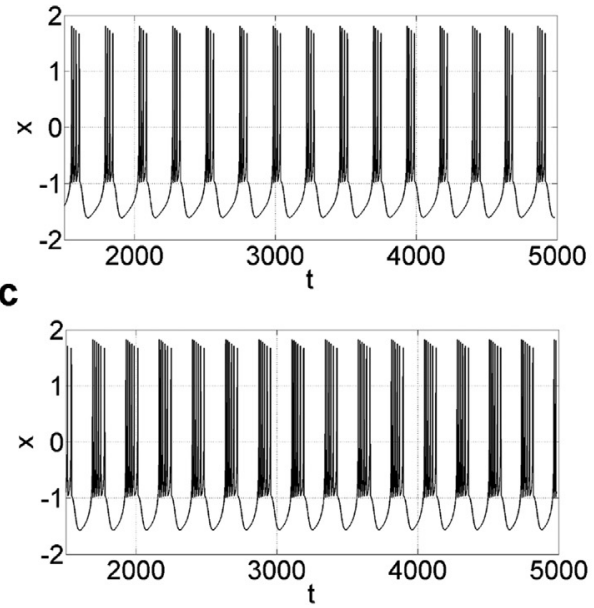

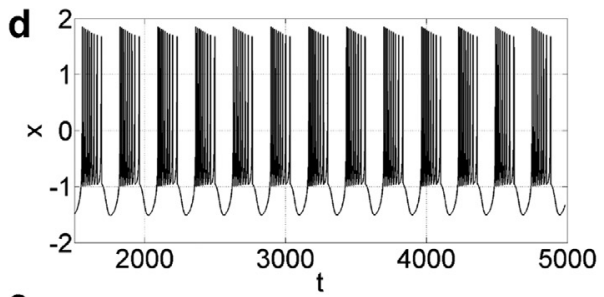

e

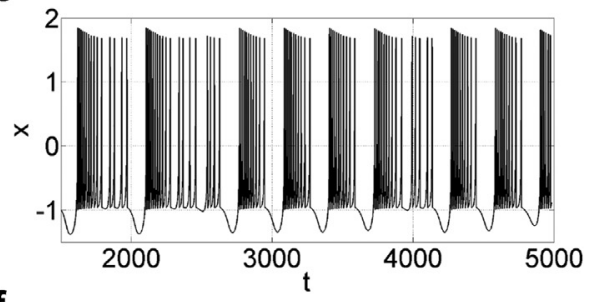

f

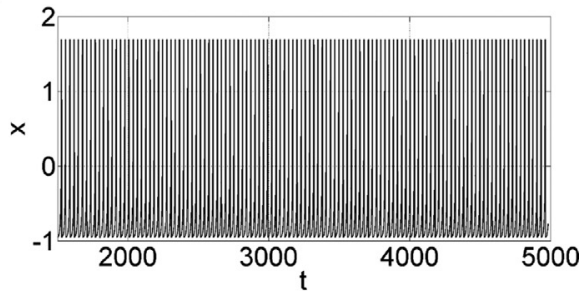

g

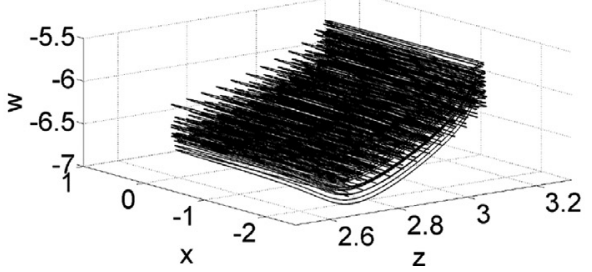

Fig. 6. Time evolution of the dynamical variable $x(t)$ of the extended HR model for different values of the applied current: $(\mathrm{a}) I_{D C}=1.01$; (b) $I_{D C}=1.426$; (c) $I_{D C}=2.03$; (d) $I_{D C}=2.64$; (e) $I_{D C}=3.0249$; (f) $I_{D C}=3.431$. (g) The corresponding three dimensional phase diagram ( $x, z$, $w$ ).

EC) phenomenon are illustrated. There exist regions where the chaotic motion is rapidly destroyed as the control parameter passes through a value called critical crisis value. This phenomenon is known as exterior crisis or blue sky catastrophe [57,63-66]. In other regions of the control parameter, the crisis creates rather than destroys a chaotic attractor. We assist at the sudden increase of the chaotic attractor, as the afore mentioned parameter is varied through its critical value [57,63-66]. Here, the chaotic attractor collides with an unstable orbit within its bassin of attraction. For example, Fig. 8 illustrates crisisinduced intermittency from time series for the extended Hindmarsh-Rose neuronal oscillator. Just before the crisis (Fig. 8(a)), the orbit cycles through a periodic band. For $v$ values above 0.034916 , we see that for long stretches the orbit remains in a periodic band, but sometimes it bursts out and then returns to periodic behavior (Fig. 8(b) and (c)). Based on the analysis of Grebogi et al., we determine to have intermittent bursting describing the second kind of crisis liable to appear in a dynamical system [63].
In addition, when a minimum number of conditions are satisfied, the system exhibits a reverse-period doubling scenario as presented in Fig. 7(e), when $\mu$ is considered as a control parameter. The bifurcation diagrams are in perfect arrangement with the corresponding maximal Lyapunov spectrum. The number of spikes within a burst decreases until the bursting motion of the neuron disappears, as $\mu$ or $v$ becomes larger, to let the spiking motion arise. This confirms the existence of such complex behaviors in the extended HR neuron model. The variation of $\mu$ or $v$ can have two interpretations. Firstly, different values of both parameters can be seen as the consideration of different neurons with different densities of slow ion channels in their axon membrane. Taking into account the fact that neurons can be of different types in a network, a spread range of variation for both parameters ( $\mu$ or $v$ ), is relevant and useful to understand the variability between individual cells. Secondly, the consideration of the existence of chemically activated or deactivated ionic channels represents another reason for changing these parameters, in such a way 


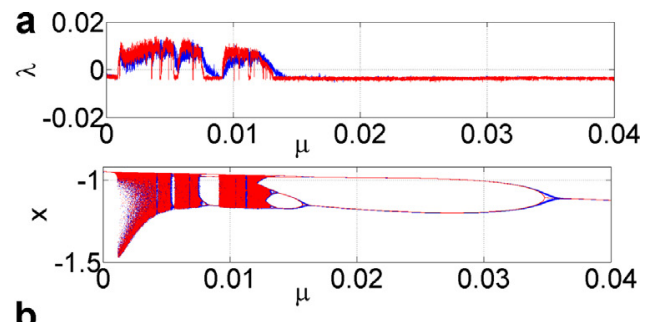

b

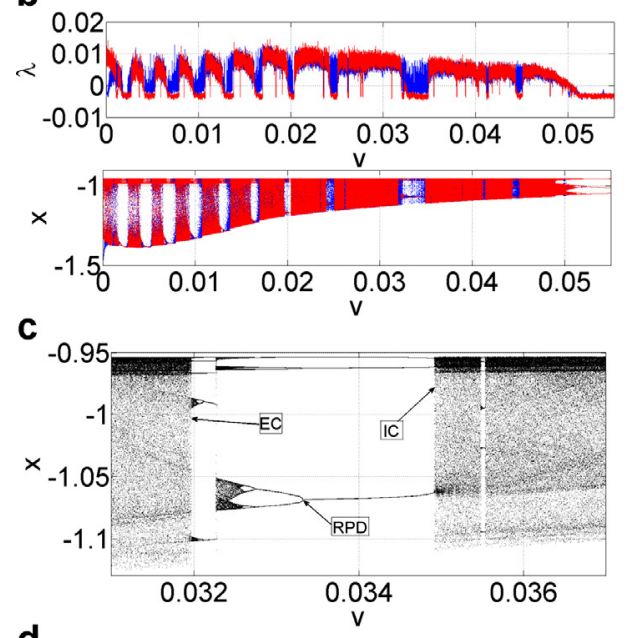

d

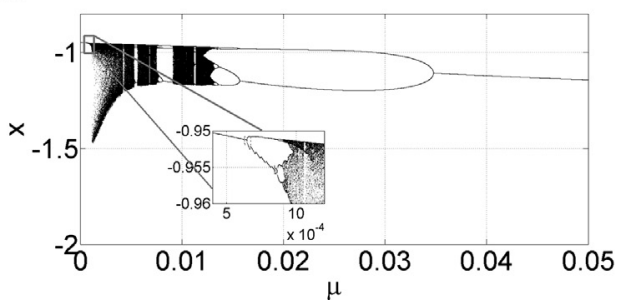

Fig. 7. Bifurcation diagrams in (a) $(\mu, x)$ and (b) $(v, x)$ planes respectively with $I_{D C}=3.0249$ and the other parameters described in the text. (c) and (d), enlargement of (a) and (b) respectively, showing reverse period doubling (RPD), exterior crisis (EC) and interior crisis (IC), (e) reverse perioddoubling illustration. (For interpretation of the references to color in this figure legend, the reader is referred to the web version of this article.)

that the permeability of the axon membrane can be modified.

\subsection{Coexistence of multiple attractors}

One of the most striking phenomenon in nonlinear dynamics is the coexistence of multiple attractors, which has been found in many systems such as electronic circuits, $[2,5,67,68]$ lasers [69] and biological systems [36,41-43,58]. Concerning the extended Hindmarsh-Rose neuronal system, effects of the initial conditions on its behavior have been shown above during numerical analysis (see Fig. 7(a) and (b)). We found that this system is sensitive to changes in initial conditions. For this purpose, these bifurcation diagrams (Fig. 7(a) and (b)), can be used as confirmation proof. We observe that the two superimposed diagrams are completely different even if they are plotted for the same range of parameters.

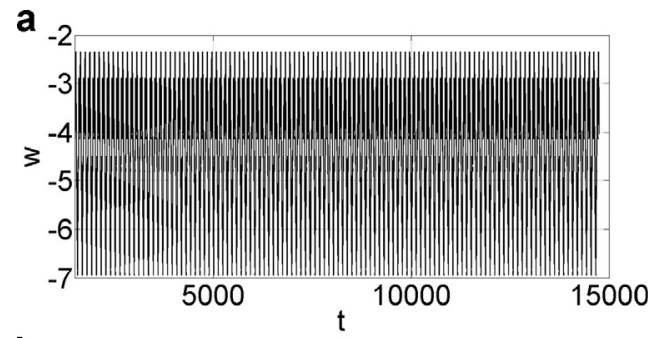

b
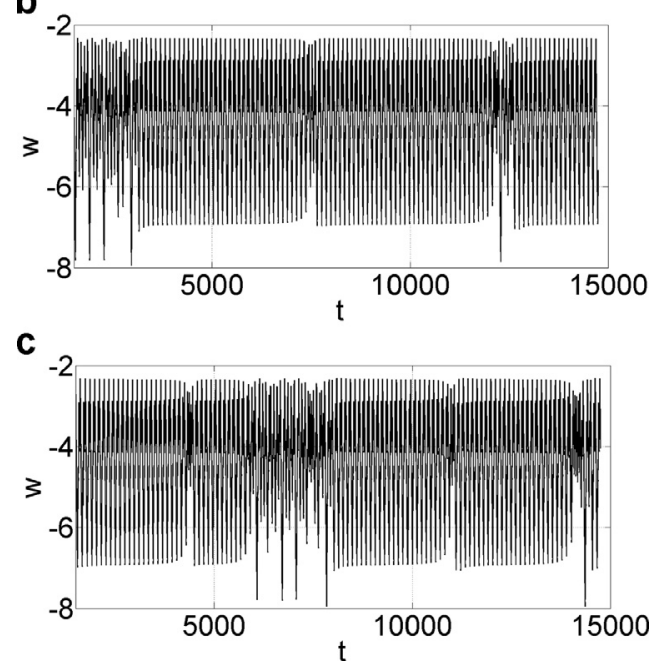

Fig. 8. Time evolution $w(t)$ near the interior crisis, completing the period-3 window for (a) $v=0.034907<v_{c}$, (b) $v=0.034917>v_{c}$, (c) $v=$ $0.034922>v_{c}$.

As it has been found by many researchers, multistability represents an essential inherent property of the dynamics of neurons and of neural networks [46-49]. It is important in understanding short-term memory and other neuronal behaviors. Nevertheless, one knows that this behavior is not wanted when a certain system performance is desired.

\section{Conclusion}

In this paper, we have presented a study of bifurcations and multistability of solutions of the extended HindmarshRose neuronal oscillator, in a wide and meaningful region of its parameters space. The system's equilibria and their stability are presented by analytical, graphical and numerical analysis (Figs. 1-3). Choosing an appropriate bifurcation parameter, we show that Hopf bifurcations also appears in this model when the bifurcation parameter approaches its critical value (see Section 3). With the help of the Hassard algorithm, we have analyzed in detail, the direction of the afore bifurcation and the stability of the bifurcating periodic solutions. We ended with numerical simulations to observe this bifurcation and to illustrate theoretical results (Fig. 4). The period doubling cascade, the crisis and the reverse period-doubling transitions to chaos have been observed, from different bifurcation diagrams and the associated largest Lyapunov exponent spectra (Figs. 5 and 7). Furthermore, the areas where the dynamics is very complex (here we have periodic spiking, 
periodic bursting and chaotic firings of spikes and bursts), are delimited (Fig. 5).

Systematic behaviors such as block structure dynamics and sharp transitions from periodic behaviors to the above complexity, have also been shown in these regions. We have seen that these complex behaviors are always present when one of the system's parameters $\left(I_{D C}, \mu\right.$ or $\left.v\right)$ is chosen as the control parameter (Figs. 5 and7). Hence, the conclusion that such behaviors are intrinsic fundamental properties of the Hindmarsh-Rose model, [28,59] can also be made for the extended one. The observed block structured dynamics and the continuous interior crisis have also been observed in the Chay neuronal model [13] and in the modified Hodgkin-Huxley model [12].

Furthermore, we have seen that the system is sensitive to change of initial conditions. This gives birth to the well known phenomenon of bistability or coexistence of attractors as described in Section 4.2. It is clear that multistability has important implications for information processing and dynamical memory in a neuron [46-49]. It seems to be a major mechanism of operation in the area of motor control, particularly in the operation of multifunctional central pattern generators.

In conclusion, the obtained results furnish potentially useful information for enhancing our knowledge on the way by which the neuronal system works, and encodes information [46-49]. For example, possible mechanisms to highlight how a nervous system give rapid response to stimulus are described by the abrupt changes observed in the system's dynamics named, the Hopf bifurcation and the crisis scenarios. Furthermore, multistability remains an additional mechanism to highlight description for such quick responses, as proposed by Foss and Milton [72]. Gonzales-Miranda [28,62] also suggested that blocks structured dynamics help to understand how neuronal coding of information evolves. Some unknown dynamical behaviors still remain in this system. Moreover, one knows that multistability is not desirable in other applications. In these cases, some control is necessary to track one of the two attractors depending of the desired application. Treatment of these questions remains a subject of future studies and is under investigation.

\section{Acknowledgments}

HAC thanks the Abdus Salam International Centre for Theoretical Physics for hospitality. PLF acknowledges support by Grant no. 2014/13272-1 São Paulo Research Foundation (FAPESP). HAC and PLF thank the ICTP-SAIFR (Fapesp 2011//11973-4) for their support.

\section{Appendix A. Some mathematical expressions}

Here we investigate some mathematical expressions obtained during calculus. First, we gives the constants obtained during the application of the Routh-Hurwitz criterion for the stability of the system.

$$
\begin{aligned}
\bar{A}= & -(1+k v) \vartheta^{2}+\left[2 f x_{e} a+k^{2} v^{2}+2 k v(1+s d)\right. \\
& +2 s d+1] \vartheta-\left[k v\left(1+2 s d+s^{2} d^{2}\right)+\operatorname{vrg}(1+k v)\right. \\
& \left.+(1+s d)\left(k^{2} v^{2}-s d\right)+2 f x_{e} a(s d-1)\right],
\end{aligned}
$$

$$
\begin{aligned}
\bar{B}= & (1+k v) \vartheta^{3}-\left[2 f x_{e} a+2 k v(2-s d)+2 k^{2} v^{2}\right. \\
& +2 s d+2] \vartheta^{2}+\left[2 k^{2} v^{2}(2+s d)+2 f x_{e} a(k v+s d+2)\right. \\
& +\left(v r g+s^{2} d^{2}\right)(1+k v)+4 k v(1+s d)+k^{3} v^{3} \\
& +2 s d+1] \vartheta-\left[2 f x_{e} a(1+k v s d+k v)\right. \\
& +\left(k^{3} v^{3}+k v\right)(1+s d)+k^{2} v^{2}(2+s d) \\
& \left.+\operatorname{vrg}(1-2 k v s d+2 k v-2 s d)+s d+k^{2} v^{3} r g\right],
\end{aligned}
$$

$$
\begin{aligned}
\bar{C}= & {[1+k v(2+k v)] \vartheta^{3}-\left[k^{3} v^{3}+k^{2} v^{2}(s d+4)\right.} \\
& \left.+(1+k v)\left(4 f x_{e} a+v r g\right)+2 k v(2+s d)+s d+1\right] \vartheta^{2} \\
& +\left[2 f x_{e} a\left(v r g+2 s d k v+k^{2} v^{2}+4 k v+2+s d\right)\right. \\
& +k^{2} v^{2}(4+s d)+v r g\left(2-2 k v s d+2 k^{2} v^{2}+4 k v-2 s d\right) \\
& \left.+k^{3} v^{3}(2+s d)+s d(1+k v)+4\left(f x_{e}\right)^{2} a^{2}+2 k v\right] \vartheta \\
& -\left[2 f x_{e} a\left(v^{2} r g k+2 k v+2 v r g+k^{2} v^{2}+k v s d+s d\right)\right. \\
& +k^{2} v^{2}+k^{3} v^{3}+v r g\left(k v^{2} r g+2 k^{2} v^{2}+2 v k+v r g\right) \\
& \left.+4\left(f x_{e}\right)^{2} a^{2}\right],
\end{aligned}
$$

and

$$
\begin{aligned}
\bar{D}= & (k v+v r g)(1+k v) \vartheta^{3}-\left[2 f x_{e} a\left(v r g+k^{2} v^{2}+2 k v\right)\right. \\
& \left.+k^{3} v^{3}+v r g\left(2 k v+k^{2} v^{2}-1\right)+2 k^{2} v^{2}+k v\right] \vartheta^{2} \\
& +\left[2 f x_{e} a\left(v r g+2 k v+k^{3} v^{3}+2 k^{2} v^{2}\right)+v r g(v r g+2 k v\right. \\
& \left.\left.+k v^{2} r g+2 k^{2} v^{2}\right)+k^{3} v^{3}+k^{2} v^{2}+4 f x_{e}^{2} a^{2} k v\right] \vartheta \\
& -\left[2 f x_{e} a\left(v^{2} r g k+k^{2} v r g+k^{3} v^{3}-k^{2} v^{2}\right)+4 f x_{e}^{2} a^{2} k v\right] .
\end{aligned}
$$

Furthermore, the eigenvectors of the system corresponding to the eigenvalues described in Section 3 are respectively:

$$
\begin{aligned}
v_{1}= & \left(1, \frac{i \omega_{0}}{a}\left(1-\frac{w}{i \omega_{0}}+\frac{d \mu s}{i \omega_{0}\left(i \omega_{0}+\mu\right)}\right), \frac{\mu s}{i \omega_{0}+\mu},\right. \\
& \left.-\frac{i \omega_{0} v r}{a\left(i \omega_{0}-k v\right)}\left(1-\frac{w}{i \omega_{0}}+\frac{d \mu s}{i \omega_{0}\left(i \omega_{0}+\mu\right)}\right)\right),
\end{aligned}
$$

$$
\begin{aligned}
v_{2}= & \left(1,-\frac{i \omega_{0}}{a}\left(1+\frac{w}{i \omega_{0}}-\frac{d \mu s}{i \omega_{0}\left(i \omega_{0}+\mu\right)}\right), \frac{\mu s}{\mu-i \omega_{0}},\right. \\
& \left.-\frac{i \omega_{0} v r}{a\left(i \omega_{0}+k v\right)}\left(1+\frac{w}{i \omega_{0}}-\frac{d \mu s}{i \omega_{0}\left(\mu-i \omega_{0}\right)}\right)\right),
\end{aligned}
$$

$$
\begin{aligned}
v_{3}= & \left(1, \frac{\varsigma_{3}}{a}\left(1-\frac{w}{\varsigma_{3}}+\frac{d \mu s}{\varsigma_{3}\left(\varsigma_{3}+\mu\right)}\right), \frac{\mu s}{\varsigma_{3}+\mu},\right. \\
& \left.-\frac{\varsigma_{3} v r}{a\left(\varsigma_{3}-k v\right)}\left(1-\frac{w}{\varsigma_{3}}+\frac{d \mu s}{\varsigma_{3}\left(\varsigma_{3}+\mu\right)}\right)\right),
\end{aligned}
$$

and

$$
\begin{aligned}
v_{4}= & \left(1, \frac{\varsigma_{4}}{a}\left(1-\frac{w}{\varsigma_{4}}+\frac{d \mu s}{\varsigma_{4}\left(\varsigma_{4}+\mu\right)}\right), \frac{\mu s}{\varsigma_{4}+\mu},\right. \\
& \left.-\frac{\varsigma_{4} v r}{a\left(\varsigma_{4}-k v\right)}\left(1-\frac{w}{\varsigma_{4}}+\frac{d \mu s}{\varsigma_{4}\left(\varsigma_{4}+\mu\right)}\right)\right) .
\end{aligned}
$$


The numerical expression of the similarity transformation $P$ and its inverse $P^{-1}$ are described by:

$P=\left(\begin{array}{cccc}1.0 & 0 & 1.0 & 1.0 \\ 7.258414757 & 1.510428036 & 10.206001501 & -1.189478134 \\ 1.025009947 & 1.736244239 & 4.003535743 & -0.0673813137 \\ 0.0199520742 & -0.0939322452 & 13.673064908 & -0.0004359258\end{array}\right)$,

and

$P^{-1}=\left(\begin{array}{cccc}0.15142991 & 0.1341805554 & -0.1208326264 & -0.0758512541 \\ -0.055123825 & -0.0826223578 & 0.6412292351 & -0.1220513791 \\ -0.0005725916 & -0.0007676583 & 0.004585192 & 0.0724088079 \\ 0.8491426815 & -0.1334128971 & 0.1162474336 & 0.0034424462\end{array}\right)$.

\section{References}

[1] Lorentz EN. Deterministic non-periodic flows. J Atmos Sci 1963;20:130-41.

[2] Liu L, Su Y, Liu C, Liu T. A modified Lorentz system. Int J Nonlinear Sci 2006;7:187-91.

[3] Rössler OE. An equation for continuous chaos. Phys Lett A 1976;57:397-8.

[4] Chen G, Ueta T. Yet another chaotic attractor. Int J Bifurc Chaos 1999;9:1456.

[5] Kengne J, Chedjou JC, Kom M, Kyamakya K, Kamdoum VT. Regular oscillations, chaos and multistability in a system of two coupled van der Pol oscillators: numerical and experimental studies. Nonlinear Dyn 2014;76:1119-32.

[6] Lu J, Chen G. A new chaotic attractor coined. Int J Bifurc Chaos 2002;12:659-61.

[7] Qi G, Du S, Chen G, Chen Z, Yuang Z. On a four-dimensional chaotic system. Chaos Solitons Fractals 2005a;23:1671-82.

[8] Qi G, Chen G, Du S, Chen Z, Yuang Z. Analysis of a new chaotic system. Physica A 2005b;352:295-308.

[9] Hodgkin AL, Huxley AF. A quantitative description of membrane current and its application to conduction and excitation in nerve. J Physiol 1952;117:500-44.

[10] Bowong S. A new adaptive chaos synchronization principle for a class of chaotic systems. Int J Nonlinear Sci 2005;6:399-408.

[11] Brown HA, Huber MT, Dewald M, Schafer K, Voigt K. Computer simulations of neuronal signal transduction: The role of nonlinear dynamics and noise. Int J Bifurc Chaos 1998;8:881-9.

[12] Chay TR. Chaos in a three-variable model of an excitable cell. Physica D 1985;16:233-42.

[13] Chen G, Dong X. From chaos to order: methodologies, perspectives and applications. Singapore: World Scientific; 1998.

[14] Sparrow C. The Lorentz equations: bifurcation, chaos and strange attractors. New York: Springer-Verlag; 1982.

[15] Hodgkin AL. The local electric changes associated with repetitive action in a non-medullated axon. J Physiol 1948;107:165-81.

[16] Rinzel J, Ermentrout GB. Analysis of neural excitability and oscillations.. Methods Neuronal Model 1998;2:251-91.

[17] Selverston AI, Rabinovich MI, Abarbanel HDI, Elson R, Szucs A, Pinto RD, et al. Reliable circuits from irregular neurons: A dynamical approach to understanding central pattern generators. J Physiol 2000;94:357-74.

[18] Izhikevich EM. Which model to use for cortical spiking neurons. IEEE Trans Neuron Net 2004;15:1063-70.

[19] Izhikevich EM. Dynamical systems in neuroscience. Cambridge: MIT Press; 2007.

[20] Morris C, Lecar H. Voltage oscillations in the barnacle giant muscle fiber. Biophys J 1981;35:193-213.

[21] Hindmarsh JL, Rose RM. A model of neuronal bursting using three coupled first order differential equations. R Soc B Biol Sci 1984;221:87-102.

[22] Hindmarsh JL, Rose RM. A model of the nerve impulse using two first-order differential equations. Nat Lond 1982;296:162-4.

[23] Pinto RD, Varona P, Valkovskii AR, Szucs A, Abarbanel HDI, Rabinovich MI. Synchronous behavior of two coupled electronic neurons. Phys Rev E 2000;62:2644.

[24] Coombes S, Bressloff PC. Bursting: The genesis of rhythm in the nervous system. London: World Scientific; 2012.

[25] De Lange E, Hasler M. Predicting single spikes and spike patterns with the Hindmarsh-Rose model. Biol Cybern 2008;99:349-60.

[26] Marco S, Daniele L, De Lange E. The Hindmarsh-Rose neuron model: Bifurcation analysis and piecewise-linear approximations. Chaos 2008; 18:03312810.

[27] Gonzalez-Miranda JM. Complex bifurcation structures in the Hindmarsh-Rose model.. Int J Bifurc Chaos 2007;17:3071-83.
[28] Gonzalez-Miranda JM. Observation of a continuous interior crisis in the Hindmarsh-Rose neuron model. Chaos 2003;13:825-45.

[29] Megam Ngouonkadi EB, Fotsin HB, Louodop Fotso PH. The combined effect of dynamic chemical and electrical synapses in time-delayinduced phase-transition to synchrony in coupled bursting neurons. Int J Bifurc Chaos 2014;24:1-16.

[30] Hassard BD, Kazarinoff ND, Wan YH. Theory and applications of Hopf bifurcation. Cambridge University Press; 1981.

[31] Arena P, Fortuna L, Frasca M, La Rosa M. Locally active HindmarshRose neurons. Chaos Solitons Fractals 2006;27:405-12.

[32] Wang W, Perez G, Cerdeira HA. Dynamical behavior of the firings in a coupled neuronal system. Phys Rev E 1993;47:2893-8.

[33] Hansel D, Sompolinsky H. Synchronization and computation in a chaotic neural network. Phys Rev Lett 1992;68:718-21.

[34] Duan LX, Lu QS. Codimension-two bifurcation in the slow-fast Hindmarsh-Rose model with two parameters. Chin Phys Lett 2005;22:1325-8.

[35] Innocenti G, Morelli A, Genesio R, Torcini A. Dynamical phases of the Hindmarsh-Rose neuronal model: studies of the transition from bursting to spiking chaos. Chaos 2007;17:04312811.

[36] Innocenti G, Genesio R. On the dynamics of chaotic spiking-bursting transition in the Hindmarsh-Rose neuron. Chaos 2009;19:0231241-8.

[37] Fakcle M, Huerta R, Rabinovich MI, Abarbanel HDI, Elson RC, Selverston AI. Modeling observed chaotic oscillations in bursting neurons: the role of calcium dynamics and IP3. Biol Cybern 2000;82:517-27.

[38] Rabinovich MI, Pinto RD, Abarbanel HDI, Trumer E, Stiestberg G, Huerta R, et al. Recovery of hidden information through synaptic dynamics. Net Comput Neural Syst 2002;13:487-501.

[39] Arecchi FT, Meucci R, Puccioni G, Tredicce J. Experimental evidence of subharmonic bifurcations, multistability and turbulence in a qswitched gas laser. Phys Rev Lett 1982;49:1217.

[40] Feudel U. Complex dynamics in multistable systems. Int J Bifurc Chaos 2008;18:1607-26.

[41] Marin B, Barnett WH, Doloc-Mihu A, Calabrese RL, Cymbalyuk GS. High prevalence of multistability of rest states and bursting in a database of a model neuron.. PLoS Comput Biol 2013;9(3):112.E1002930.

[42] Shilnikov A, Cymbalyuk G. Transition between tonic spiking and bursting in a neuron model via the blue-sky catastrophe. Phys Rev Lett 2005;94:1-4.048101.

[43] Malashchenko T, Shilnikov A, Cymbalyuk G. Bistability of bursting and silence regimes in a model of a leech heart interneuron. Phys Rev E 2011a;84:1-8.041910.

[44] Pastor-Diaz I, Lopez-Fragas A. Dynamics of two coupled van der Pol oscillators. Phys Rev E 1995;52:1480-9.

[45] Pastor-Diaz I, Perez-Garcia VM, Encinas-Sanz F, Gerra JM. Ordered and chaotic behavior of two coupled van der Pol oscillators. Phys Rev E 1983;48:171-82.

[46] Heyward P, Ennis M, Keller A, Shipley MT. Membrane bistability in olfactory bulb mitral cells. J Neurosci 2001;21:5311-20.

[47] Hounsgaard J, Kiehn O. Serotonin-induced bistability of turtle motoneurones caused by a nifedipine-sensitive calcium plateau potential. J Physiol 1989;414:265-82.

[48] Loewenstein Y, Mahon S, Chadderton P, Kitamura K. Sompolinsky h. bistability of cerebellar purkinje cells modulated by sensory stimulation. Nat Neurosci 2005;8:202-11.

[49] Perrier JF, Hounsgaard J. Development and regulation of response properties in spinal cord motoneurons. Brain Res Bull 2000;53:52935.

[50] Fröhlich F, Bazhenov M. Coexistence of tonic firing and bursting in cortical neurons. Phys Rev E 2006;74:031922.

[51] Gutkin BS, Jost J, Tuckwell HC. Inhibition of rhythmic neural spiking by noise: the occurrence of a minimum in activity with increasing noise. Naturwissenschaften 2009;96:1091-7.

[52] Guttman R, Lewis S, Rinzel J. Control of repetitive firing in squid axon membrane as a model for a neurone oscillator. J Physiol 1980;305:377-95

[53] Malashchenko T, Shilnikov A, Cymbalyuk G. Six types of multistability in a neuronal model based on slow calcium current. PloS ONE 2011b;6:e21782

[54] Rinzel J. On repetitive activity in nerve. Nlm NIH 1978;37:2793-802.

[55] Shilnikov A, Calabrese R, Cymbalyuk G. Mechanism of bi-stability: tonic spiking and bursting in a neuron model. Phys Rev E 2005;71:19.

[56] Chua LO, Wu CW, Huang Q, Zhong GA. A universal circuit for studying and generating chaos-part i: Routes to chaos. IEEE Trans Circuit Syst I Fund Theory Appl 1993;40:731-44. 
[57] Grebogi C, Ott E, Yoke JA. Crises, sudden changes in chaotic attractors, and transient chaos. Physica D 1983;7:181-200.

[58] Cushing JM, Henson SM, Blackburn CC. Multiple mixed attractors in a competition model. J Biol Dyn 2007:1:347-62.

[59] Hassard BD. Bifurcation of periodic solutions of the Hodgkin-Huxley model for squid giant axon. J Theor Biol 1978;71:401-20.

[60] Guckenheimer J, Holmes P. Nonlinear oscillations, dynamical systems and bifurcations of vector field. New York: Springer; 1983.

[61] Wolf A, Swift JB, Swinney HL, Vastano JA. Determining Lyapunov exponents from a time series. Physica D 1985;16:285-317.

[62] Gonzalez-Miranda JM. Block structured dynamics and neuronal coding. Phys Rev E 2005;72:051922.

[63] Grebogi C, Ott E, Romeiras F, Yoke JA. Critical exponents for crisisinduced intermittency. Phys Rev A 1987a;36:5365-80.

[64] Abraham RH, Stewart HB. A chaotic blue sky catastrophe in forced relaxation oscillations. Physica D 1986;21:394-400.

[65] Grebogi C, Ott E, Yorke JA. Chaos, strange attractors, and fractal basin boundaries in nonlinear dynamics. Sci New Ser 1987b;238:632-8.
[66] Nayfeh AH, Balachandran B. Applied nonlinear dynamics analytical, computational, and experimental methods. Germany: Wiley; 1995.

[67] Maggio GM, De Feo O, Kennedy MP. Nonlinear analysis of the Colpitts oscillator and application to design. IEEE Trans Circuits Syst 1999;46:1118-30

[68] Vaithianathan V, Veijun J. Coexistence of four different attractors in a fundamental power system model. IEEE Trans Circuits Syst I 1999;46:405-9.

[69] Masoller C. Coexistence of attractors in a laser diode with optical feedback from a large external cavity. Phys Rev A 1994;50:2569-78.

[70] Zhou X, Wu Y, Li Y, Wei Z. Hopf bifurcation of the Liu system. Chaos Solitons Fractals 2008;36:1385-91.

[71] Hassard BD, Wan YH. Bifurcation formulae derived from center manifold theory. J Math Anal Appl 1978;63:297-312.

[72] Foss J, Milton J. Multistability in recurrent neural loops arising from delay. J Neurophysiol 2000;84:975-85. 